\title{
Finite 1-Regular Cayley Graphs of Valency 5
}

\author{
Jing Jian Li, ${ }^{1,2}$ Ben Gong Lou, ${ }^{1}$ and Xiao Jun Zhang ${ }^{2,3}$ \\ ${ }^{1}$ School of Mathematics and Statistics, Yunnan University, Kunming, Yunnan 650031, China \\ ${ }^{2}$ School of Mathematics and Information Sciences, Guangxi University, Nanning 530004, China \\ ${ }^{3}$ School of Computer Science and Engineering, University of Electronic Science and Technology of China, Chengdu 611731, China
}

Correspondence should be addressed to Ben Gong Lou; bengong188@163.com

Received 15 November 2012; Revised 11 January 2013; Accepted 28 February 2013

Academic Editor: Cai Heng Li

Copyright (C) 2013 Jing Jian Li et al. This is an open access article distributed under the Creative Commons Attribution License, which permits unrestricted use, distribution, and reproduction in any medium, provided the original work is properly cited.

Let $\Gamma=\operatorname{Cay}(G, S)$ and $G \leq X \leq \operatorname{Aut} \Gamma$. We say $\Gamma$ is $(X, 1)$-regular Cayley graph if $X$ acts regularly on its arcs. $\Gamma$ is said to be corefree if $G$ is core-free in some $X \leq \operatorname{Aut}(\operatorname{Cay}(G, S))$. In this paper, we prove that if an $(X, 1)$-regular Cayley graph of valency 5 is not normal or binormal, then it is the normal cover of one of two core-free ones up to isomorphism. In particular, there are no core-free 1 -regular Cayley graphs of valency 5.

\section{Introduction}

We assume that all graphs in this paper are finite, simple, and undirected.

Let $\Gamma$ be a graph. Denote the vertex set, arc set, and full automorphism group of $\Gamma$ by $V \Gamma, A \Gamma$, and Aut $\Gamma$, respectively. A graph $\Gamma$ is called $X$-vertex-transitive or $X$-arc-transitive if $X$ acts transitively on $V \Gamma$ or $A \Gamma$, where $X \leq A u t \Gamma$. $\Gamma$ is simply called vertex-transitive, arc-transitive for the case where $X=$ Aut $\Gamma$. In particular, $\Gamma$ is called $(X, 1)$-regular if $X \leq A u t \Gamma$ acts regularly on its arcs and then 1-regular when $X=$ Aut $\Gamma$.

Let $G$ be a finite group with identity element 1 . For a subset $S$ of $G$ with $1 \notin S=S^{-1}:=\left\{x^{-1} \mid x \in S\right\}$, the Cayley graph $\operatorname{Cay}(G, S)$ of $G$ (with respect to $S$ ) is defined as the graph with vertex set $G$ such that $x, y \in G$ are adjacent if and only if $y x^{-1} \in S$. It is easy to see that a Cayley graph $\operatorname{Cay}(G, S)$ has valency $|S|$, and it is connected if and only if $\langle S\rangle=G$.

Li proved in [1] that there are only finite number of core-free $s$-transitive Cayley graphs of valency $k$ for $s \in$ $\{2,3,4,5,7\}$ and $k \geq 3$ and that, with the exceptions $s=$ 2 and $(s, k)=(3,7)$, every $s$-transitive Cayley graph is a normal cover of a core-free one. It was proved in [2] that there are 15 core-free $s$-transitive cubic Cayley graphs up to isomorphism, and there are no core-free 1-regular cubic Cayley graphs. A natural problem arises. Characterize 1transitive Cayley graphs, in particular, which graphs are 1regular? Until now, the result about 1-regular graphs mainly focused constructing examples. For example, Frucht gave the first example of cubic 1-regular graph in [3]. After then, Conder and Praeger constructed two infinite families of cubic 1-regular graphs in [4]. Marušič [5] and Malnič et al. [6] constructed two infinite families of tetravalent 1-regular graphs. Classifying such graphs has aroused great interest. Motivated by above results and problem, we consider 1regular Cayley graphs of valency 5 in this paper.

A graph $\Gamma$ can be viewed as a Cayley graph of a group $G$ if and only if Aut $\Gamma$ contains a subgroup that is isomorphic to $G$ and acts regularly on the vertex set. For convenience, we denote this regular subgroup still by $G$. If $X \leq A u t \Gamma$ contains a normal subgroup that is regular and isomorphic to $G$, then $\Gamma$ is called an $X$-normal Cayley graph of $G$; if $G$ is not normal in $X$ but has a subgroup which is normal in $X$ and semiregular on $V \Gamma$ with exactly two orbits, then $\Gamma$ is called an $X$-bi-normal Cayley graph; furthermore if $X=\operatorname{Aut} \Gamma, \Gamma$ is called normal or bi-normal. Some characterization of normal and bi-normal Cayley graphs has given in $[1,2]$.

For a Cayley graph $\Gamma=\operatorname{Cay}(G, S), \Gamma$ is said to be core-free (with respect to $G$ ) if $G$ is core-free in some $X \leq A u t \Gamma$; that is, $\operatorname{Core}_{X}(G)=\cap_{x \in X} G^{x}=1$.

The main result of this paper is the following assertion.

Theorem 1. Let $\Gamma=\operatorname{Cay}(G, S)$ be an $(X, 1)$-regular Cayley graph of valency 5 , where $G \leq X \leq \operatorname{Aut} \Gamma$. Let $n(G)$ be the number of nonisomorphic core-free $(X, 1)$-regular Cayley 
TABLE 1

\begin{tabular}{lccccc}
\hline Number & $X$ & $G$ & $n(G)$ & 1-regular & Remark \\
\hline 1 & $\mathrm{~A}_{5}$ & $\mathrm{~A}_{4}$ & 1 & No & Icosahedron \\
2 & $\mathrm{~S}_{5}$ & $\mathrm{~S}_{4}$ & 1 & No & \\
\hline
\end{tabular}

graph of valency 5 with the regular subgroup equal to $G$. Then either

(i) $\Gamma$ is an X-normal or X-bi-normal Cayley graph or

(ii) $\Gamma$ is a nontrivial normal cover of one line of Table 1.

In particular, there are no core-free 1-regular Cayley graphs of valency 5 . ately.

By Theorem 1, we can get the following remark immedi-

Remark 2. Let $\Gamma=\operatorname{Cay}(G, S)$ be an 1-regular Cayley graph of valency 5 . Then $\Gamma$ is normal or bi-normal.

\section{Examples}

In this section we give some examples of graphs appearing in Theorem 1.

Example 3. Let $M=\langle a\rangle \cong \mathbb{Z}_{11}$ be a cyclic group. Assume that $\tau \in \operatorname{Aut}(M)$ is of order 10 and $X=M:\langle\tau\rangle \cong \mathbb{Z}_{11}: \mathbb{Z}_{10}$. Let

$$
G=M:\left\langle\tau^{5}\right\rangle \cong \mathrm{D}_{22}
$$

Suppose that

$$
S=g^{\left\langle\tau^{2}\right\rangle}=\left\{g, g^{\tau^{2}}, g^{\tau^{4}}, g^{\tau^{6}}, g^{\tau^{8}}\right\}
$$

where $g \in G$ is an involution such that $g \neq \tau^{5}$. Let $\Gamma=$ Cay $(G, S)$ be the Cayley graph of the dihedral group $G$ with respect to $S$. Then $\Gamma$ is a connected $(X, 1)$-regular Cayley graph of valency 5 . In particular, $\Gamma$ is $X$-normal.

Proof. Let

$$
G=\langle a\rangle:\langle b\rangle=\left\{1, a, a^{2}, \ldots, a^{10}, b, a b, a^{2} b, \ldots, a^{10} b\right\} \cong \mathrm{D}_{22},
$$

where $b=\tau^{5}$.

Noting $o(a)=11$, we may assume that $a^{\tau}=a^{9}$. Since the involution $g \in G$ is not equal to $b$, we may let $g=a^{i} b$ for some $1 \leq i<11$ such that $(9, i)=1$. Then $g^{\tau^{2}}=\left(a^{i} b\right)^{\tau^{2}}=a^{81 i} b=$ $a^{4 i} b$, and so $g^{\tau^{2}} g^{-1}=a^{3 i} \in\left\langle g^{\left\langle\tau^{2}\right\rangle}\right\rangle=\langle S\rangle$. Thus the element $g^{\tau^{2}} g^{-1}$ is of order 11 as $(3 i, 11)=1$. So $\langle S\rangle=\left\langle g^{\left\langle\tau^{2}\right\rangle}\right\rangle=G$; that is, $\Gamma=\operatorname{Cay}(G, S)$ is connected.

Obviously, $G \triangleleft X \leq \operatorname{Aut} \Gamma$ and $X_{1}=\left\langle\tau^{2}\right\rangle$. However, $|X|=$ $55=|A \Gamma|$; then $\Gamma$ is an $(X, 1)$-regular normal Cayley graph of $G$ of valency 5 .
Example 4. Let $G=\left\langle a, b \mid a^{5}=b^{2}=1, a^{b}=a^{-1}\right\rangle \cong \mathrm{D}_{10}$. Set $S=\left\{b, a b, a^{2} b, a^{3} b, a^{4} b\right\}$ and $\Gamma=\operatorname{Cay}(G, S)$. Then $\Gamma \cong \mathrm{K}_{5,5}$ and Aut $\Gamma=S_{5} 2 S_{2}$. Let $X=\left(\mathbb{Z}_{5} \times \mathbb{Z}_{5}\right): \mathbb{Z}_{2} \not \mathrm{D}_{10} \times \mathbb{Z}_{5}$ such that $G \leq X \leq$ Aut $\Gamma$. It follows that $\operatorname{Core}_{X}(G) \cong \mathbb{Z}_{5}$. Then $X_{\alpha} \cong \mathbb{Z}_{5}$ for $\alpha \in V \Gamma$, and furthermore $\Gamma$ is $(X, 1)$-regular. Obviously $G$ is not normal in $X$. However, Core $_{X}(G) \unlhd X$ is semiregular and has exactly two orbits on $V \Gamma$; then $\Gamma$ is an $(X, 1)$-regular Cayley graph of valency 5 . In particular, $\Gamma$ is $X$-bi-normal.

\section{The Proof of Main Results}

In this section, we will prove our main results. We first present some properties about normal Cayley graphs.

For a Cayley graph $\Gamma=\operatorname{Cay}(G, S)$, we have a subgroup of $\operatorname{Aut}(G)$ :

$$
\operatorname{Aut}(G, S)=\left\{\sigma \in \operatorname{Aut}(G) \mid S^{\sigma}=S\right\}
$$

Clearly it is a subgroup of the stabilizer in Aut $\Gamma$ of the vertex corresponding to the identity 1 of $G$. Since $\Gamma$ is connected, $\operatorname{Aut}(G, S)$ acts faithfully on $S$. By Godsil [7, Lemma 2.1], the normalizer $\mathrm{N}_{\mathrm{Aut} \Gamma}(G)=G: \operatorname{Aut}(G, S)$. So $\Gamma=\operatorname{Cay}(G, S)$ is a normal Cayley graph if and only if $\operatorname{Aut}(G, S)=(\operatorname{Aut} \Gamma)_{1}$.

Let $\Gamma=\operatorname{Cay}(G, S)$ be an $(X, 1)$-regular Cayley graph of valency 5 such that $G \leq X \leq$ Aut $\Gamma$. Then $S$ contains at least one involution. Let $\mathrm{K}=\operatorname{Core}_{X}(G)$, which is the core of $G$ in $X$.

Lemma 5. Assume that $\mathrm{K}=1$. Then $(X, G)=\left(\mathrm{A}_{5}, \mathrm{~A}_{4}\right)$ or $\left(\mathrm{S}_{5}, \mathrm{~S}_{4}\right)$.

Proof. Let $H$ be the stabilizer in $X$ of the vertex corresponding to the identity of $G$. Then $H \cong \mathbb{Z}_{5}, H \cap G=1$, and $X=G H$. Let $[X: G]$ be the set of right cosets of $G$ in $X$. Consider the action of $X$ on $[X: G]$ by the right multiplication. Then we get that $X$ is a primitive permutation group of degree 5 and $G$ is a stabilizer of $X$. Since $\Gamma$ has valency $5,|G|=|V \Gamma| \geq 6$, and so $|X|=|G||H| \geq 30$. Then we can show $X \cong \mathrm{A}_{5}$ or $\mathrm{S}_{5}$, and then $G=\mathrm{A}_{4}$ or $\mathrm{S}_{4}$, respectively.

Lemma 6. Suppose that $G=\mathrm{A}_{4}$ and $X=\mathrm{A}_{5}$. Then $\Gamma$ is the icosahedron graph. Moreover, Aut $\Gamma=\mathrm{A}_{5} \times \mathbb{Z}_{2}$ and $\Gamma$ is not 1-regular.

Proof. Note that $X=G H$, where $X \cong \mathrm{A}_{5}, G \cong \mathrm{A}_{4}$, and $H \cong \mathbb{Z}_{5}$. Since $X$ has no nontrivial normal subgroup, $\Gamma$ is not bipartite. So $\Gamma$ is the icosahedron graph. Further by Magma [8], Aut $\Gamma=A_{5} \times \mathbb{Z}_{2}$, so $\Gamma$ is not 1-regular.

Lemma 7. Suppose that $G=\mathrm{S}_{4}$ and $X=\mathrm{S}_{5}$. Then the graph $\Gamma$ is not 1-regular and there is only one isomorphism class of these graphs.

Proof. Note that $G=\mathrm{S}_{4}, X=\mathrm{S}_{5}$, and $X=G H$. Let $H=\langle\sigma\rangle$, where $\sigma=\left(\begin{array}{llll}1 & 2 & 3 & 4\end{array}\right.$ 5). By considering the right multiplication action of $X$ on the right cosets of $G$ in $X, G$ can be viewed as a stabilizer of $X$ acting on $\{1,2,3,4,5\}$. Without lost generality, we may assume that 1 is fixed by $G$. Take an involution $\tau \in S$. Then, by [2], $\tau \in \mathrm{S}_{5} \backslash \mathrm{N}_{\mathrm{S}_{5}}(H)$ and we can identify $S$ with $H \tau H \cap G$. Note that $\tau \in G \leq S_{4}$ 
and $\mathrm{N}_{\mathrm{S}_{5}}(H)=H: \operatorname{Aut}(H)=\left\langle\left(\begin{array}{lllll}1 & 2 & 3 & 4 & 5\end{array}\right)\right\rangle:\left\langle\left(\begin{array}{llll}2 & 3 & 5 & 4\end{array}\right)\right\rangle \cong$ $\mathbb{Z}_{5}: \mathbb{Z}_{4}$; then $\tau$ is one of the following: (2 5), (3 5), (2 3), (3 4), (4 5), (2 4), (2 3)(4 5), and (2 4)(3 5). Note $H=\left\langle\left(\begin{array}{lllll}1 & 2 & 3 & 4 & 5\end{array}\right)\right\rangle$. Assume that $\tau=\left(\begin{array}{ll}2 & 5\end{array}\right)$; by calculation,

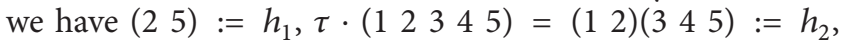
$\tau \cdot\left(\begin{array}{lllll}1 & 3 & 5 & 2 & 4\end{array}\right)=\left(\begin{array}{llll}1 & 3 & 5 & 4\end{array}\right):=h_{3}, \tau \cdot\left(\begin{array}{lllll}1 & 4 & 2 & 5 & 3\end{array}\right)=$ $\left(\begin{array}{llll}1 & 4 & 2 & 3\end{array}\right):=h_{4}$, and $\tau \cdot\left(\begin{array}{lllll}1 & 5 & 4 & 3 & 2\end{array}\right)=\left(\begin{array}{ll}1 & 5\end{array}\right)\left(\begin{array}{lll}2 & 4 & 3\end{array}\right):=h_{5}$. Then $H(25) H=\left\{H h_{1}, H h_{2}, H h_{3}, H h_{4}, H h_{5}\right\}=\{(25)$, (1 5)(2 3 4), (1 $\left.4 \begin{array}{lll} & 5 & 3\end{array}\right),($ (1 2$)\left(\begin{array}{lll}3 & 5 & 4\end{array}\right),\left(\begin{array}{llll}1 & 3 & 2 & 4\end{array}\right),\left(\begin{array}{lll}1 & 5\end{array}\right)\left(\begin{array}{lll}2 & 4 & 3\end{array}\right)$,

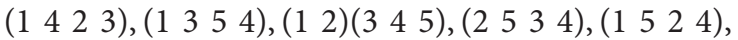

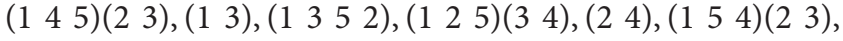
(1 2 3)(4 5), (3 5), (1 5 2) (3 4), (1 422 5), (1 4), (1 3 2)(4 5), (1 $\left.\left.\begin{array}{llll}1 & 2 & 5 & 3\end{array}\right),\left(\begin{array}{llll}2 & 4 & 3 & 5\end{array}\right)\right\}$. Thus the corresponding $S$ is

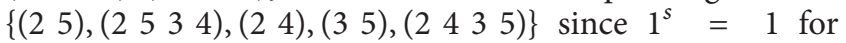
each $s \in H(25) H$. By similar argument, for every $\tau$, we can work out $S$ explicitly, which is one of the following four

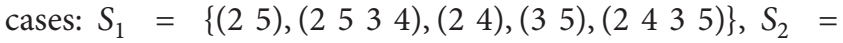
$\left\{\left(\begin{array}{ll}2 & 3\end{array}\right),\left(\begin{array}{l}3 \\ 4\end{array}\right),\left(\begin{array}{ll}4 & 5\end{array}\right),\left(\begin{array}{llll}2 & 3 & 4 & 5\end{array}\right),\left(\begin{array}{llll}2 & 5 & 4 & 3\end{array}\right)\right\}, S_{3}=\left\{\left(\begin{array}{lll}2 & 3\end{array}\right)\left(\begin{array}{l}4 \\ 4\end{array}\right)\right.$,

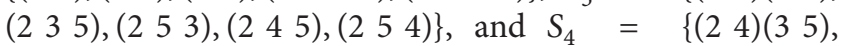
(2 4 3), (3 5 4), (2 34 4), (3 4 5 $)$ ) .

Now let $A=$ Aut $\Gamma$. We declare that $X \neq A$. Assume that $X=A$. Note that $G=\mathrm{N}_{A}(G)=G \operatorname{Aut}(G, S)$; then $\operatorname{Aut}(G, S)=$

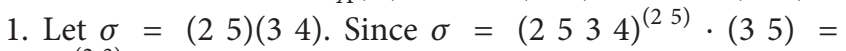

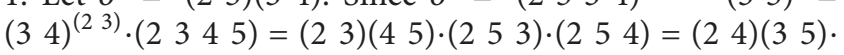
(2 4 3 3 ).(3 54 ),$\sigma \in G$ and $S^{\sigma}=S$ for any possible $S$. Therefore $\sigma \in \operatorname{Aut}(G, S)$, which leads to a contradiction. So the assertion is right; that is, $\Gamma$ is not 1-regular.

Let $G_{i}=\left\langle S_{i}\right\rangle$ and $\Gamma_{i}=\operatorname{Cay}\left(G_{i}, S_{i}\right)$ for $i \in\{1,2,3,4\}$. Set $\gamma=\left(\begin{array}{llll}2 & 4 & 5 & 3\end{array}\right)$, then $S_{1}^{\gamma}=S_{2}$ and $S_{3}^{\gamma}=S_{4}$. It follows that $G_{1}^{\gamma}=G_{2}$ and $G_{3}^{\gamma}=G_{4}$, namely, $\Gamma_{1} \cong \Gamma_{2}$ and $\Gamma_{3} \cong \Gamma_{4}$. Now we consider $G_{1}=\left\langle S_{1}\right\rangle$. Note that $(24)=$ $\left.\left(\begin{array}{ll}2 & 5\end{array}\right)^{(2435}\right)$ and $\left(\begin{array}{ll}3 & 5\end{array}\right)=\left(\begin{array}{llll}2 & 5 & 3 & 4\end{array}\right)^{(25)} \cdot\left(\begin{array}{llll}2 & 5 & 3 & 4\end{array}\right)^{2}$, then $G_{1}=$

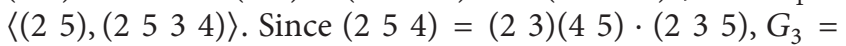
$\left\langle S_{3}\right\rangle=\left\langle\left(\begin{array}{ll}2 & 3\end{array}\right)\left(\begin{array}{ll}4 & 5\end{array}\right),\left(\begin{array}{lll}2 & 3 & 5\end{array}\right)\right\rangle$. On the other hand, $\left(\begin{array}{llll}2 & 5 & 3 & 4\end{array}\right)^{4}=$ $(25)^{2}=((25) \cdot(2534))^{3}=1$ and $\left(\begin{array}{lll}2 & 3 & 5\end{array}\right)^{3}=$ $\left(\left(\begin{array}{ll}2 & 3\end{array}\right)\left(\begin{array}{ll}4 & 5\end{array}\right)\right)^{2}=\left(\left(\begin{array}{ll}2 & 3\end{array}\right)\left(\begin{array}{lll}4 & 5\end{array}\right) \cdot\left(\begin{array}{lll}2 & 3 & 5\end{array}\right)\right)^{3}=1$, then $G_{1} \cong \mathrm{S}_{4}$ and $G_{3} \cong A_{4}$. By the assumption, $\Gamma_{3}$ is not the graph satisfying conditions. So far we get the result that there is only one isomorphism class of graphs when $G=\mathrm{S}_{4}$.

To finish our proof, we need to introduce some definitions and properties. Assume that $\Gamma$ is an $X$-vertex transitive graph with $X$ being a subgroup of Aut $\Gamma$. Let $N$ be a normal subgroup of $X$. Denote the set of $N$-orbits in $V \Gamma$ by $V_{N}$. The normal quotient $\Gamma_{N}$ of $\Gamma$ induced by $N$ is defined as the graph with vertex set $V_{N}$, and two vertices $B, C \in V_{N}$ are adjacent if there exist $u \in B$ and $v \in C$ such that they are adjacent in $\Gamma$. It is easy to show that $X / N$ acts transitively on the vertex set of $\Gamma_{N}$. Assume further that $\Gamma$ is $X$-edge-transitive. Then $X / N$ acts transitively on the edge set of $\Gamma_{N}$, and the valency $\operatorname{val}(\Gamma)=m \operatorname{val}\left(\Gamma_{N}\right)$ for some positive integer $m$. If $m=1$, then $\Gamma$ is called a normal cover of $\Gamma_{N}$.

Proof of Theorem 1. Let $\Gamma=\operatorname{Cay}(G, S)$ be an $(X, 1)$-regular Cayley graph of valency 5 , where $G \leq X \leq \operatorname{Aut} \Gamma$. Then it is trivial to see that $\Gamma$ is connected. Let $N=\operatorname{Core}_{X}(G)$ be the core of $G$ in $X$. Assume that $N$ is not trivial. Then either $G=N$ or $|G: N| \geq 2$. The former implies $G \unlhd X$; that is, $\Gamma$ is an $X$-normal Cayley graph with respect to $G$. For the case where $|G: N|=2$, it is easy to verify $\Gamma$ is an $X$-bi-normal Cayley graph. Suppose that $|G: N|>2$; namely, $N$ has at least three orbits on $V \Gamma$. Since $\operatorname{val}(\Gamma)=5$ is a prime and $\Gamma$ is $(X, 1)$-regular, $\Gamma$ is a cover of $\Gamma_{N}$ and $G / N \leq X / N \leq \operatorname{Aut}_{N}$. We have that $\Gamma_{N}$ is a Cayley graph of $G / N$ and $\Gamma_{N}$ is core-free with respect to $G / N$. Now suppose that $N$ is trivial, then $\Gamma$ is a core-free one. According to Lemmas 5, 6, and 7, there are two core-free $(X, 1)$-regular Cayley graphs of valency 5 (up to isomorphism) as in Table 1 . As far, Theorem 1 holds.

\section{Acknowledgments}

The project was sponsored by the Foundation of Guangxi University (no. XBZ110328), the Fund of Yunnan University (no. 2012CG015), NNSF (nos. 11126343, 11226141, and 11226045), and NSF of Guangxi (no. 2012GXNSFBA053010).

\section{References}

[1] C. H. Li, "Finite $s$-arc transitive Cayley graphs and flag-transitive projective planes," Proceedings of the American Mathematical Society, vol. 133, no. 1, pp. 31-41, 2005.

[2] J. J. Li and Z. P. Lu, "Cubic s-arc transitive Cayley graphs," Discrete Mathematics, vol. 309, no. 20, pp. 6014-6025, 2009.

[3] R. Frucht, "A one-regular graph of degree three," Canadian Journal of Mathematics, vol. 4, pp. 240-247, 1952.

[4] M. D. E. Conder and C. E. Praeger, "Remarks on path-transitivity in finite graphs," European Journal of Combinatorics, vol. 17, no. 4, pp. 371-378, 1996.

[5] D. Marušič, "A family of one-regular graphs of valency 4," European Journal of Combinatorics, vol. 18, no. 1, pp. 59-64, 1997.

[6] A. Malnič, D. Marušič, and N. Seifter, "Constructing infinite one-regular graphs," European Journal of Combinatorics, vol. 20, no. 8, pp. 845-853, 1999.

[7] C. D. Godsil, "On the full automorphism group of a graph," Combinatorica, vol. 1, no. 3, pp. 243-256, 1981.

[8] W. Bosma, J. Cannon, and C. Playoust, “The Magma algebra system. I. The user language," Journal of Symbolic Computation, vol. 24, no. 3-4, pp. 235-265, 1997. 


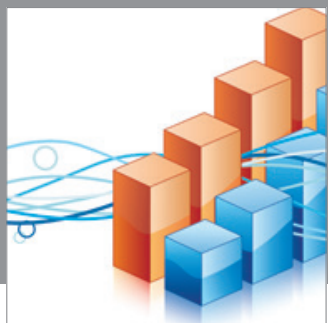

Advances in

Operations Research

mansans

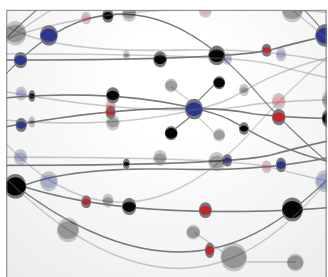

The Scientific World Journal

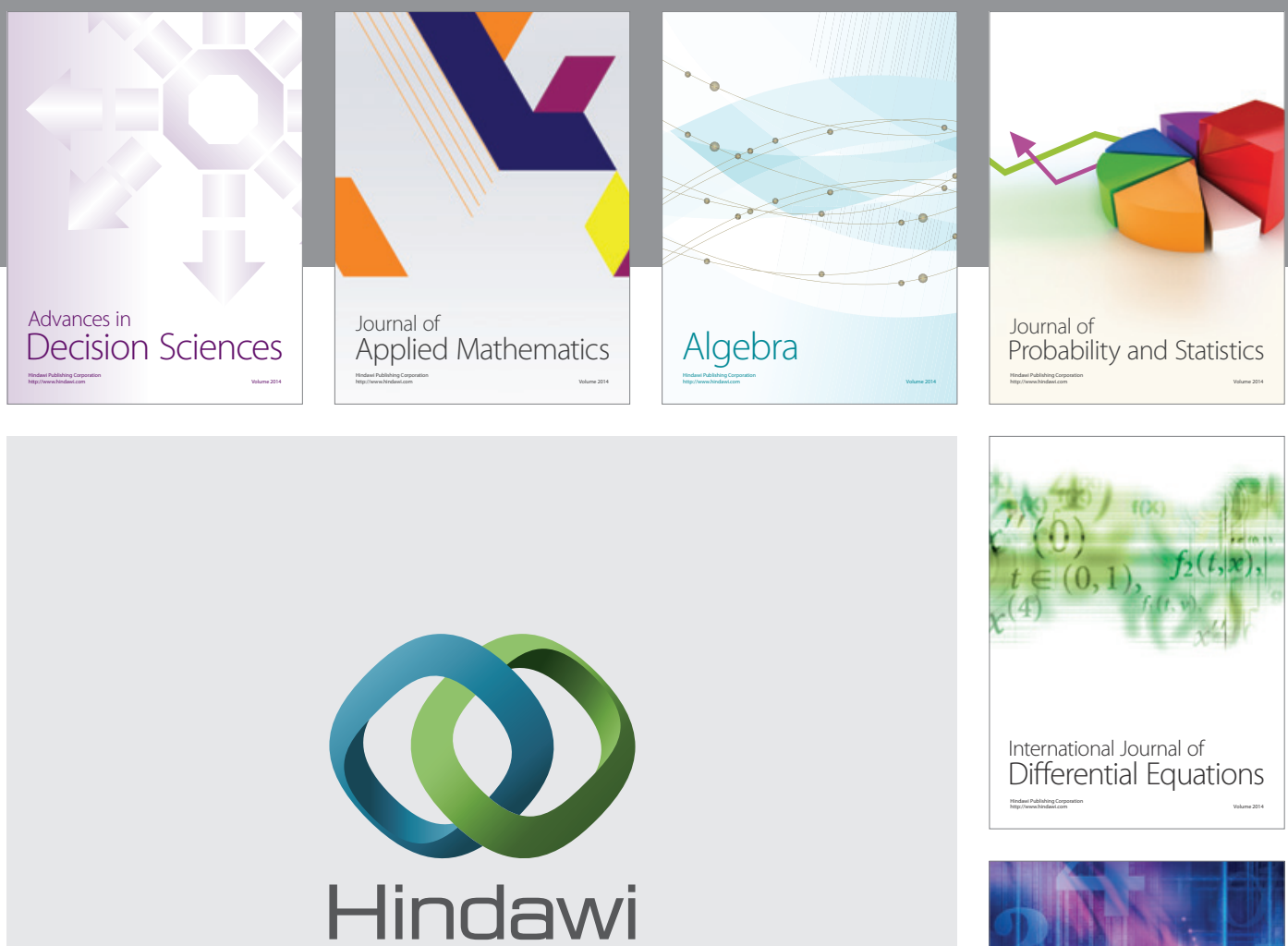

Submit your manuscripts at http://www.hindawi.com
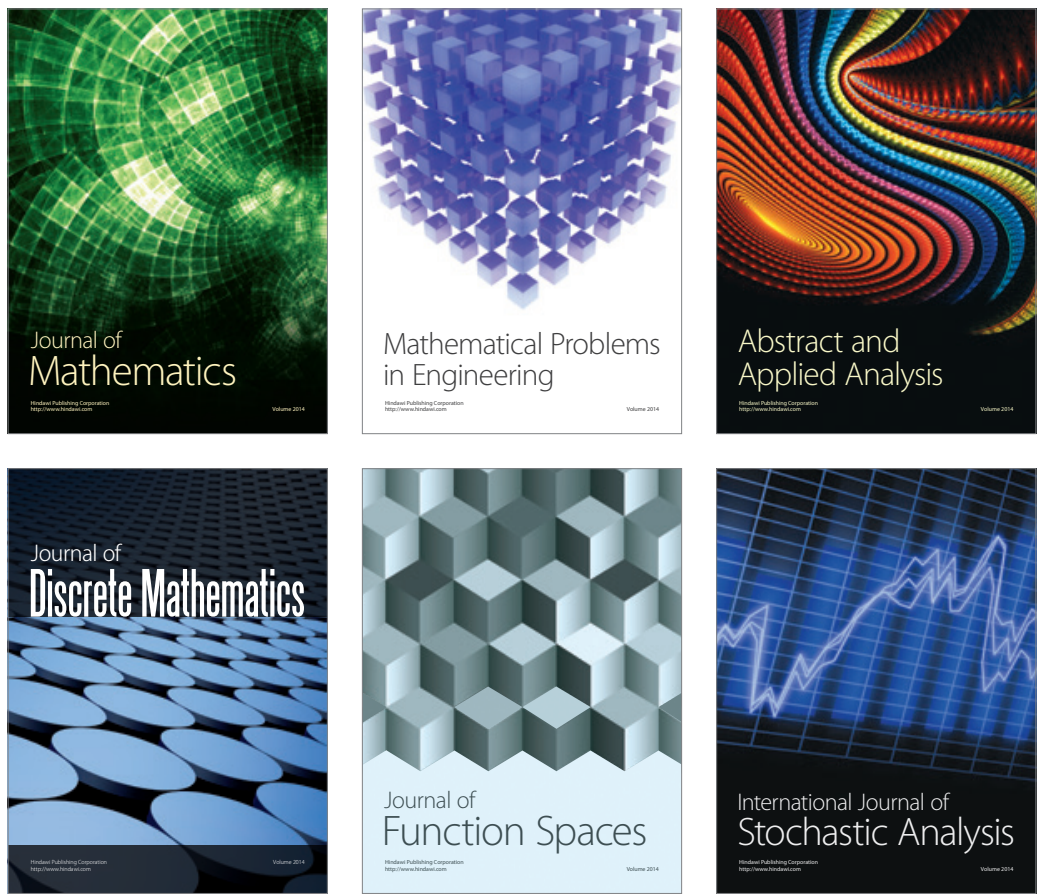

Journal of

Function Spaces

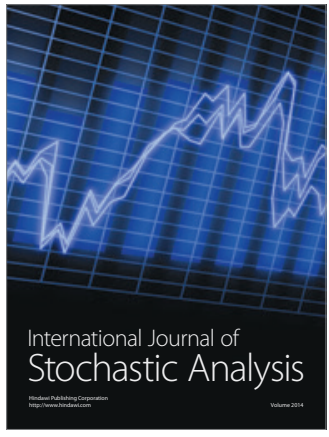

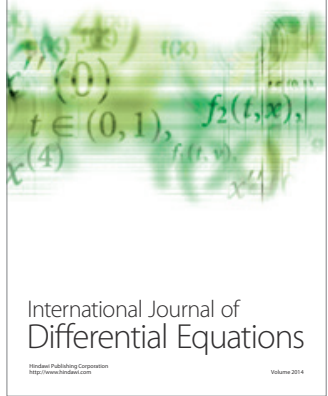
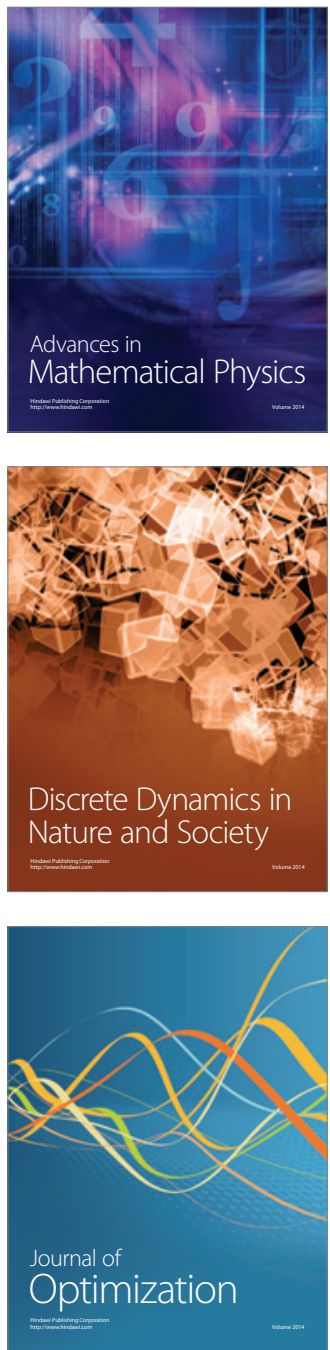